\title{
CENTRAL AND LOGARITHMIC CENTRAL IMAGE CHORD TRANSFORMATIONS FOR INVARIANT OBJECT RECOGNITION
}

\author{
Veaceslav Perju ${ }^{1 *}$, ORCID 0000-0002-7755-4277, \\ Vladislav Cojuhari², ORCID 0000-0001-7540-2184 \\ ${ }^{1}$ Agency for Military Science and Memory \\ 47, Tighina Str., Chisinau, MD2001, Republic of Moldova \\ ${ }^{2}$ Ministry of Internal Affairs \\ 75, Stefan cel Mare Bld, Chisinau, MD2012, Republic of Moldova \\ *Corresponding author: Veaceslav Perju, vlperju@yahoo.com
}

Received: 11. 28. 2020

Accepted: 01. 19. 2021

\begin{abstract}
Pattern descriptors invariant to rotation, scaling, and translation represents an important direction in the elaboration of the real time object recognition systems. In this article, the new kinds of object descriptors based on chord transformation are presented. There are described new methods of image presentation - Central and Logarithmic Central Image Chord Transformations (CICT and LCICT). It is shown that the CICToperation makes it possible to achieve invariance to object rotation. In the case of implementation of the LCICT transformation, invariance to changes in the rotation and scale of the object is achieved. The possibilities of implementing the CICTand LCICToperations are discussed. The algorithms of these operations for contour images are presented. The possibilities of integrated implementation of CICT and LCICT operations are considered. A generalized CICT operation for a full (halftone) image is defined. The structures of the coherent optical processors that implement operations of basic and integral image chord transformations are presented.
\end{abstract}

Keywords: image, chord, transformation, recognition, processor.

\section{Introduction}

Invariant Object Recognition (IOR) is of great importance for many civil and military applications and supposes the identification and classification of the object in real-time, regardless of spatial position, angular orientation, etc.

Different directions and approaches exist in IOR, such as using the support vector machines [1], deep learning techniques [2], neural networks [3], image moments feathers $[4,5]$, correlation techniques [6, 7], etc.

A pattern descriptors invariant to rotation, scaling, translation (RST) represent an important direction in IOR. In article [8] the RST invariance is obtained by applying the Fourier-Mellin transform on the radial and angular coordinates of the pattern's Radon image. In article [9] a novel descriptor is proposed based on the ring-projection and dualtree complex wavelets which permit the transformation of the pattern from a 2-D image to 
a 1-D signal. In article [10] the Hough transform realization is proposed in an incoherent optical processor.

The described above approaches in IOR need substantial computational expenditures which in many cases do not permit the realization of real-time. Also, numerical accuracy does not correspond to the standards.

One of the perspective directions in object recognition is based on the chord functions using due to their properties of invariance to the object position, rotation, or scaling changes. In the article [11] a hybrid optical-digital system for chord functions calculation is described. In article [12] an optical processor that realizes a generalized chord transformation is presented. The wedge-ring detector samples of autocorrelation are shown to be the histograms of the chord distributions. In article [13] a logarithmic chord transformation of the images (LHTI) is proposed. Structures of an optical-electronic processor for LHTI realization and of a system for object recognition are described.

In this article, the results of the development of the invariant object recognition based on the new kinds of the chord transformation are presented. Section 2 describes new methods of image transformations - Central and Logarithmic Central Image Chord Transformation (CICT and LCICT).

It is shown that the CICToperation makes it possible to achieve invariance to object rotation. In the case of implementation of the LCICTtransformation, invariance to changes in the rotation and scale of the object is achieved. In section 3 the possibilities of implementing the CICT and LCICT operations are discussed. The algorithms of these operations for contour images are presented.

The possibilities of integrated implementation of CICT and LCICT operations are considered. A generalized CICT operation for a full (halftone) image is defined. The structure of a coherent optical processor that implements operations of integral image chord transformation is presented.

\section{Central and logarithmic central image chord transformations}

Let $P(x, y)$ is an initial object (Figure 1.a) and $P_{b}(x, y)$ is the function of the object's image, which is described by the binary external contour, $P_{b}(x, y)=\{0,1\}$, and $\left(x_{c}, y_{c}\right)-$ coordinates of the center (Figure 1.b). For every pair of points $\left(x_{i}, y_{i}\right)$ and $\left(x_{k}, y_{k}\right)$ is constructed a chord $A_{i} B_{k}$ passing through the point $\left(x_{c}, y_{c}\right)$, is determined the angle $\psi_{i k}$ between chord and $X$-axis, and chord's length $\mathrm{R}_{\mathrm{ik}}$ as:

$$
\begin{aligned}
& \psi_{i k}=\operatorname{arctg}\left[\left(\mathrm{y}_{\mathrm{k}}-\mathrm{y}_{\mathrm{i}}\right) /\left(\mathrm{x}_{\mathrm{k}}-\mathrm{x}_{\mathrm{i}}\right)\right] \\
& R_{i k}=\sqrt{\left(\mathrm{x}_{\mathrm{k}}-\mathrm{x}_{\mathrm{i}}\right)^{2}+\left(\mathrm{y}_{\mathrm{k}}-\mathrm{y}_{\mathrm{i}}\right)^{2}}
\end{aligned}
$$

In this case, the object's image can be characterized by the function $H\left(\psi_{\mathrm{ik}}, R_{\mathrm{ik}}\right)$, which describes all possible chords drawn through the point $\left(\mathrm{x}_{c}, \mathrm{y}_{\mathrm{c}}\right)$. Transformation, through which was obtained the function $\mathrm{H}\left(\Psi_{\mathrm{ik}}, \mathrm{R}_{\mathrm{ik}}\right)$ will be named the Central Image Chord Transformation (CIHT):

$$
\mathrm{P}_{\mathrm{b}}(\mathrm{x}, \mathrm{y}) \rightarrow \mathrm{T}\left\{\mathrm{P}_{\mathrm{b}}(\mathrm{x}, \mathrm{y})\right\}=\mathrm{H}\left(\Psi_{\mathrm{ik}}, \mathrm{R}_{\mathrm{ik}}\right)=\mathrm{H}\left(\mathrm{x}_{1}, \mathrm{y}_{1}\right)=\mathrm{P}_{\mathrm{b}}\left(\mathrm{x}_{1}, \mathrm{y}_{1}\right),
$$

where $\mathrm{T}\{\ldots\}$ - the operation of CIHT,

$$
x_{1}=\Psi_{i k}, y_{1}=R_{i k} .
$$




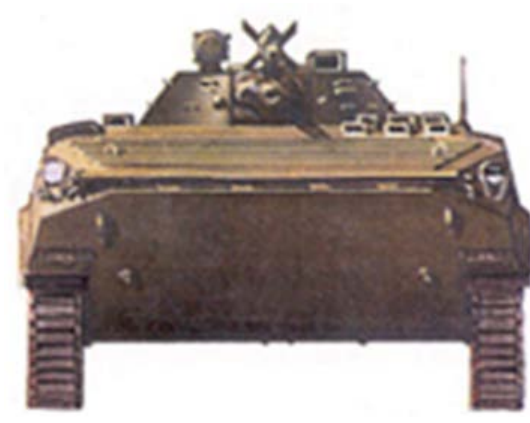

a)

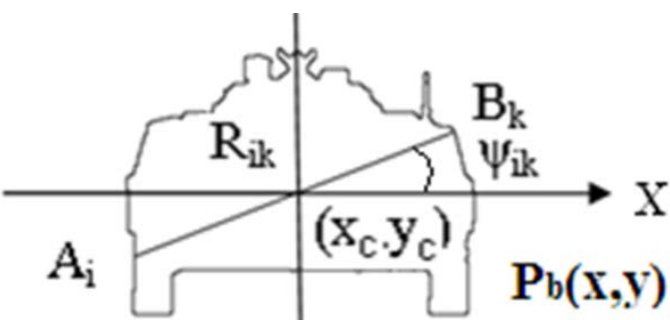

b)

Figure 1. Initial (a) and countered (b) objects.

Let's introduce the definition of Logarithmic Central Image Chord Transformation ( $\mathrm{LCICT}$ ). This transformation is realizing by taking the logarithm of the parameter $\mathrm{R}_{\mathrm{ik}}$ of the function $\mathrm{H}\left(\Psi_{\mathrm{ik},}, \mathrm{R}_{\mathrm{ik}}\right)$ :

$$
\operatorname{LT}\left\{H\left(\Psi_{i k}, R_{i k}\right)\right\}=L H\left(\Psi_{i k}, \ln R_{i k}\right)=L H\left(\Psi_{i k}, W_{i k}\right)=P_{b}\left(x_{2}, y_{2}\right)
$$

where $L T\{\ldots\}$ - the operation of LCICT,

$$
\mathrm{x}_{2}=\Psi_{\mathrm{ik}}, \mathrm{y}_{2}=\mathrm{w}_{\mathrm{ik}}=\ln \mathrm{R}_{\mathrm{ik}}
$$

In Figure 2 are presented the transformations $\mathrm{CICT}(\mathrm{a})$ and $\mathrm{LCICT}(\mathrm{b})$. It is evident, that the rotation of the initial image will shift the function $\mathrm{R}(\psi)$ along the axis $\psi$, i.e. the CICToperation allows to achieve invariance to the rotation of the object. In the case of the LCICTtransformation, the object scale change will shift the function $\ln R(\psi)$ along the axis $\mathrm{LnR}$, i.e. this transformation allows to achieve the invariance to the scale change.

\section{Possibilities of the image chord transformation's realization}

Let's consider the possibilities of the CICT and LCICT operations realization. Let the input image contain an object $P\left(x^{\prime}, y^{\prime}\right)$ located in the area of interest's center with coordinates $\left(\mathrm{x}_{c}, \mathrm{y}_{\mathrm{c}}\right)$. The function $\mathrm{P}\left(\mathrm{x}^{\prime}, \mathrm{y}^{\prime}\right)$ is converted to a binary image or represented by its external binary contour:

$$
P\left(x^{\prime}, y^{\prime}\right) \rightarrow P_{b}(x, y),
$$

where $P_{b}(x, y)=\{0,1\}$.

Then, for an angle $\psi$ varying within $0-180^{\circ}$, the chords are constructed, passing through the point $\left(x_{c}, y_{c}\right)$, their lengths $R_{i k}$ are determined, and the functions $H\left(\Psi_{i k}, R_{i k}\right)=P_{b}\left(x_{1}, y_{1}\right), \operatorname{LH}\left(\psi_{i k}, W_{i k}\right)=P_{b}\left(x_{2}, y_{2}\right)$ are formed. The described operations of CICTand LCICTcan be realized in the software or in digital signal processors, as well as in the specialized optical processors.

\subsection{Optical processor for image chord transformation}

The processor contains (Figure 3) a coherent radiation source 1, an image input module 2, a matrix of threshold optrons 3 , a photodetector 4 , and a controller 5 . The image input module 2 is an electro-optical spatial light modulator (SLM) that operates in the light transmission mode. The matrix of threshold optrons 3 performs the image binarization operation. Photodetector 4 realizing radial (output A) and logarithmic radial (output B) image scanning. Controller 5 synchronizes the operation of processor units, data storage, and communication with external devices. 


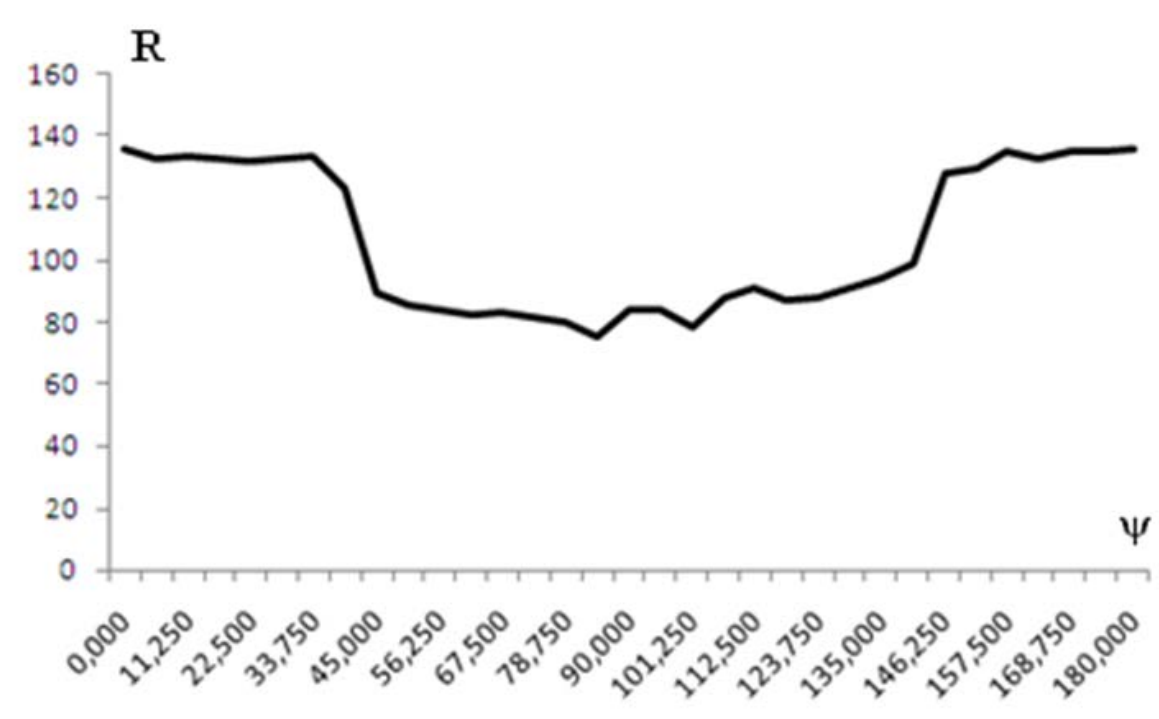

a)

$\operatorname{LnR}$

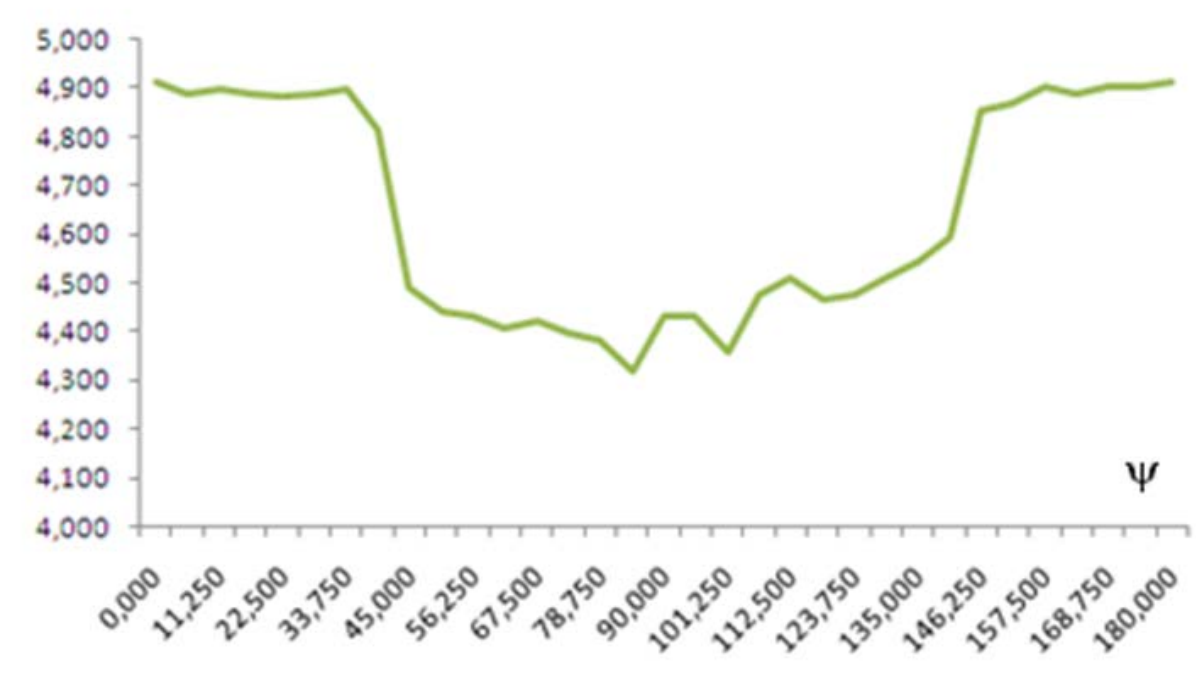

b)

Figure 2. Transformations $\mathrm{CICT}(\mathrm{a})$, and $\mathrm{LCICT}(\mathrm{b})$.

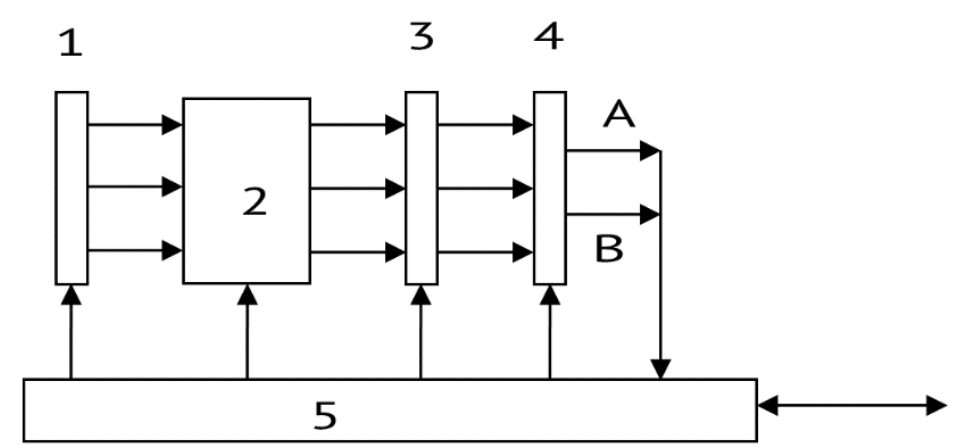

Figure 3. Optical processor for image chord transformation.

The processor functions as follows: centered image of the object $P\left(x^{\prime}, y^{\prime}\right)$ is recorded on a SLM 2. Then switch on the radiation source 1, the light beam from the output of which passes through the SLM 2, is modulated by the intensity function $P\left(x^{\prime}, y^{\prime}\right)$ and flows through the matrix of optrons 3 . As a result, the function $P\left(x^{\prime}, y^{\prime}\right)$ is binarized: $P\left(x^{\prime}, y^{\prime}\right) \rightarrow P_{b}(x, y)$ and 
then goes to the photodetector matrix 4, at the outputs $A$ and $B$ of which the transformations CICT and LCICT are formed respectively. The corresponding signal values are entered into controller 5 .

\subsection{Integral realization of CICTand LCICToperations}

The integral realization of chord transformations CICT and LCICT assumes the following. Because the values of the function $P_{b}(x, y)$ in boundary points of the object are defined as $\mathrm{Pb}_{b}(\mathrm{x}, \mathrm{y})=1$, the chord will exist between the two image points if they are a boundary, i.e.

$$
G\left(x_{i}, y_{i}, \psi_{i k}, R_{i k}\right)=P_{b}\left(x_{i}, y_{i}\right) P_{b}\left[x_{i}+R_{i k} \cos \psi_{i k}, y_{i}+R_{i k} \sin \psi_{i k}\right]=1
$$

At various values of the points' combinations on the external contour of the object, the operation CICTwill be determined as follows:

$$
\begin{aligned}
& H\left(\psi_{i k}, R_{i k}\right)=\iint G\left(x_{i}, y_{i}, \psi_{i k}, R_{i k}\right) d x d y= \\
& \iint P_{b}\left(x_{i}, y_{i}\right) P_{b}\left[x_{i}+R_{i k} \cos \psi_{i k}, y_{i}+R_{i k} \sin \psi_{i k}\right] d x d y \\
& =\iint P_{b}\left(x_{i}, y_{i}\right) P_{b}\left[x_{i}+\xi_{i k}, y_{i}+\eta_{i k}\right] d x d y=H\left(\xi_{i k}, \eta_{i k}\right)=P_{b}(x, y) * P_{b}(x, y)
\end{aligned}
$$

where $\xi_{i k}=R_{i k} \cos \psi_{i k}, \eta_{i k}=R_{i k} \sin \psi_{i k}$

Thus, the CICToperation can be implemented by calculating the autocorrelation function of the contour image $P_{b}(x, y)$. For a full (halftone) image $P(x, y)$, the generalized CICT operation will be defined as:

$$
H(\psi, R)=H(\xi, \eta)=\iint P(x, y) P[x+\xi, y+\eta] d x d y=P(x, y) * P(x, y)
$$

Let's define the generalized LCICToperation as:

$$
L H(\psi, W)=C T\{H(\xi, \eta)\}=H\left[\xi^{\prime}, \eta^{\prime}\right]
$$

where $\mathrm{CT}\{\ldots\}$ is the coordinate transformation operation,

$$
\xi^{\prime}=\operatorname{arctg}(\eta / \xi), \eta^{\prime}=\left[\ln \left(\xi^{2}+\eta^{2}\right)\right] / 2
$$

Let's show that the generalized LCICT operation is invariant to shift, change in the angular orientation and scale of the image. Let the input image be described by the function:

$$
P\left(x^{\prime}, y^{\prime}\right)=P\left[x+e_{3}, y+e_{4}, e_{1}, e_{2}\right),
$$

where $e_{1}, e_{2}$ - change of scale, the angular orientation of the object, respectively; $e_{3}, e_{4}-$ parameters of the object's displacement. The calculation of the autocorrelation functions leads to the elimination of the influence of parameters $e_{3}, e_{4}$. Really,

$$
\begin{aligned}
& H(\xi, \eta)=\iint P\left(x^{\prime}, y^{\prime}\right) P\left(x^{\prime}+\xi, y^{\prime}+\eta\right] d x^{\prime} d y^{\prime}= \\
& =F T^{-1}\left\{F T\left[P\left(x^{\prime}, y^{\prime}\right)\right] F T^{*}\left[F T P\left(x^{\prime}, y^{\prime}\right)\right]\right\}= \\
& =F T^{-1}\left\{\exp \left[j\left(u e_{3}+v e_{4}\right)\right] P\left(u^{\prime}, v^{\prime}\right) \exp \left[-j\left(u e_{3}+v e_{4}\right) P^{*}\left(u^{\prime}, v^{\prime}\right)\right\}=\right. \\
& =F T^{-1}\left\{P\left(u^{\prime}, v^{\prime}\right) P^{*}\left(u^{\prime}, v^{\prime}\right)\right\}=H\left(\xi_{1}^{\prime}, \eta_{1}^{\prime}\right),
\end{aligned}
$$


where $\mathrm{FT}, \mathrm{FT}^{-1}, \mathrm{FT}^{*}$ are the direct, inverse, and complex conjugate Fourier transform, respectively. Values $\xi_{1}^{\prime}, \eta_{1}^{\prime}$ are functions of parameters $e_{1}, e_{2}: \xi_{1}^{\prime}=F_{1}\left(e_{1}, e_{2}\right), \eta_{1}^{\prime}=F_{2}\left(e_{1}, e_{2}\right)$.

Image transformation, made by (13) allows separating the influence of the scale e1 and rotation e2 parameters of the object and reducing them to equivalent shifts:

$$
H^{\prime}(\psi, W)=C T\left\{H^{\prime}\left(\xi_{1}^{\prime}, \eta_{1}^{\prime}\right)\right\}=H_{o}\left\{\xi+\xi_{o}\left(e_{2}\right), \eta+\eta_{o}\left(e_{1}\right)\right\},
$$

where $\xi_{o}\left(e_{2}\right) \eta_{o}\left(e_{1}\right)$ are constant.

Thus, as a result of the LCICT operation, invariance to shifts, changes in the scale, and angular orientation of the object are achieved. This will allow reducing significantly the volume of computing costs in the subsequent stages of digital processing.

In Figure 4 is shown the structure of a coherent optical processor that implements operations of integral image chord transformation. The processor contains a coherent radiation source 1, an optical image input module 2, electro-optical SLMs 3 and 4, a Fourier lens 5 , a photodetector 6 , and a controller 7. The SLM 3 is used for recording the input image.

The SLM 4 is used for displaying the functions $\Phi(\xi, n)$ at the stage of optical realization of image transformation to logarithmic polar (15) or polar (16) coordinate system:

$$
\begin{aligned}
& \Phi_{l p}(\xi, \eta)=\left\{\begin{array}{lrr}
\left.\Phi_{+}(\xi, \eta)=(\xi / 2) \ln \left(\xi^{2}+\eta^{2}\right)+\xi\right] & \text { at } & \xi<0 \\
\Phi_{-}(\xi, \eta)=\Phi_{+}(\xi, \eta)-\left(2 \pi^{2} / \lambda f_{L}\right) \eta & \text { at } & \xi>0
\end{array}\right. \\
& \Phi_{p}(\xi, \eta)=\left\{\begin{array}{lrl}
\left.\Phi_{+}(\xi, \eta)=\xi\left(\xi^{2}+\eta^{2}\right)+\xi\right] & \text { at } & \xi<0 \\
\Phi_{-}(\xi, \eta)=\Phi_{+}(\xi, \eta)-\left(2 \pi^{2} / \lambda f_{L}\right) \eta & \text { at } & \xi>0
\end{array}\right.
\end{aligned}
$$

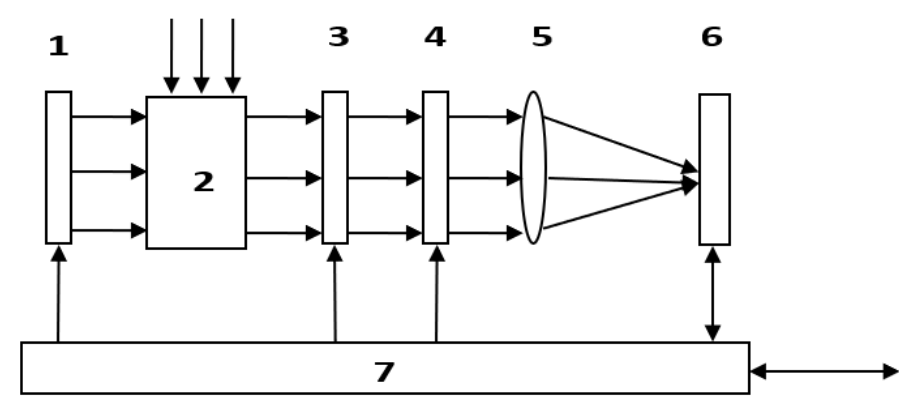

Figure 4. Optical processor for integral image chord transformation.

In expressions (15), (16) $\lambda$ - the wavelength of the radiation source $1 ; f_{L}$ - the focal length of the Fourier lens 5 . For the function $\Phi(\xi, n)$, recorded on SLM 4 and described by the expression (15) or (16), the processor will implement the LCICT or CICT operation, respectively. Module 2 has two optical inputs and one optical output. The photodetector 6 is designed to scan the optical Fourier transform formed by lens 5 and subsequent data input into the controller 7.

The processor function in the next mode. In the initial state, the input image $P\left(x^{\prime}, y^{\prime}\right)$ is recorded on the SLM 3, and the SLM 4 is transparent. Then the source of radiation 1 is switched on, the collimated light beam from the output of which goes through module 2 , 
SLM 3, is modulated by the image function $P\left(x^{\prime}, y^{\prime}\right)$ and then passing through the SLM 4. Lens 5 performs a two-dimensional Fourier transform of the image $P\left(x^{\prime}, y^{\prime}\right)$ :

$$
F T\left\{P\left(x^{\prime}, y^{\prime}\right)\right\}=\exp \left[j\left(u^{\prime} e_{3}+v^{\prime} e_{4}\right)\right] P\left(u^{\prime}, v^{\prime}\right)
$$

where $u^{\prime}=F_{3}\left(e_{1}, e_{2}\right), v^{\prime}=F_{4}\left(e_{1}, e_{2}\right)$.

The optical distribution (17) is reflected in the photodetector 6 as a Fourier spectrum:

$$
\begin{aligned}
& P_{s}\left(u^{\prime}, v^{\prime}\right)=\left|F T\left\{P\left(x^{\prime}, y^{\prime}\right)\right\}\right|^{2}=F T\left\{P\left(x^{\prime}, y^{\prime}\right)\right\} F T^{*}\left\{P\left(x^{\prime}, y^{\prime}\right)\right\}= \\
& =\exp \left[j\left(u^{\prime} e_{3}+v^{\prime} e_{4}\right)\right] P\left(u^{\prime}, v^{\prime}\right) \exp \left[-j\left(u^{\prime} e_{3}+v^{\prime} e_{4}\right) * P\left(u^{\prime}, v^{\prime}\right)=\right. \\
& =P\left(u^{\prime}, v^{\prime}\right) P^{*}\left(u^{\prime}, v^{\prime}\right) .
\end{aligned}
$$

Next, the distribution (18) is scanned by the photodetector 6 and recorded on the SLM 3 through the controller 7. Using the lens 5, the Fourier transform of the function (18) is performed, as a result of which will be obtained an optical distribution in the plane of the photodetector 6 , described as follows:

$$
\begin{aligned}
& F T\left\{P\left(u^{\prime}, v^{\prime}\right) P^{*}\left(u^{\prime}, v^{\prime}\right)\right\} .=F T\left\{F T\left[P\left(x_{1}^{\prime}, y_{1}^{\prime}\right)\right]\right\} F T\left\{F T^{*}\left[P\left(x_{1}^{\prime}, y_{1}^{\prime}\right)\right]\right\}= \\
& =F T\left\{F T\left[P\left(x_{1}^{\prime}, y_{1}^{\prime}\right)\right]\right\}^{*} F T\left\{F T\left[P\left(-x_{1}^{\prime},-y_{1}^{\prime}\right)\right]\right\}=P\left(x_{1}^{\prime}, y_{1}^{\prime}\right) * P\left(x_{1}^{\prime}, y_{1}^{\prime}\right)
\end{aligned}
$$

where ${ }^{*}$ is the correlation operation, $x_{1}^{\prime}=F_{3}\left(e_{1}, e_{2}\right), y_{1}^{\prime}=F_{4}\left(e_{1}, e_{2}\right)$.

The distribution (19) represents an autocorrelation function of the input image:

$$
\left|P\left(\xi^{\prime}, \eta^{\prime}\right)\right|^{2}=\left|P\left(x_{1}^{\prime}, y_{1}^{\prime}\right) * P\left(x_{1}^{\prime}, y_{1}^{\prime}\right)\right|^{2} .
$$

As a result, the influence of parameters $e_{3}, e_{4}$ was eliminated. The optical distribution described by the function (20) is scanned by the photodetector 6 and written to the SLM 3 via the controller 7. By this time, on the SLM 4, the function is written by the expression (15) or (16), which allows multiplying this function with the function from the SLM 3. As a result, at the output of SLM 4, the optical distribution will be characterized by the function: $P(\xi, \eta)=\left|P\left(\xi^{\prime}, \eta^{\prime}\right)\right| \exp [j \Phi(\xi, \eta)]$. After the Fourier transform realized using lens 5 , an optical distribution will be formed in the plane of the photodetector 6 :

$$
F T\{|P(\xi, \eta)| \exp [j \Phi(\xi, \eta)]\}=H(\psi, G),
$$

where $\mathrm{G}=w=\left[\ln \left[\left(\xi^{\prime}\right)^{2}+\left(\eta^{\prime}\right)^{2}\right]\right] / 2, \psi=\operatorname{arctg}(\xi / \eta)$ in the case of $\Phi(\xi, \eta)=\Phi_{l p}(\xi, \eta)$, and $\mathrm{G}=\mathrm{R}=$ $\left[\left(\xi^{\prime}\right)^{2}+\left(\eta^{\prime}\right)^{2}\right]^{1 / 2}, \psi=\operatorname{arctg}(\xi / \eta)$ in the case of $\Phi(\xi, \eta)=\Phi_{p}(\xi, \eta)$

After the function $\mathrm{H}(\Psi, \mathrm{G})$ scanning by the photodetector 6 in columns and rows, the functions $H(\Psi), H(G)$ will be formed, carrying information about the features of the input image:

$$
H_{i}(\psi)=\int_{0}^{L_{y}} H(\psi, G) l_{r} d l_{r},
$$




$$
H_{j}(G)=\int_{0}^{L_{h}} H(\psi, G) l_{h} d l_{h}
$$

where $L_{v}, L_{h}$ are the lengths of the horizontal and vertical electrodes.

From expressions (22) and (23) it follows that the function $H(\psi)$ does not depend on $G$, i.e. is invariant to change the input image scale; the function $H(G)$ is invariant to change the angular orientation of the image. At the output of the photodetector $6,2 n$ signals are generated, which is equivalent to representing the image by a vector $\mathbf{v}$ of length $D P=2 n$. Thus, the processor output generates a set of electrical signals that characterize the features of the input image.

\section{Conclusions}

Two new types of image transformations are proposed - the Central and Logarithmic Central Image Chord Transformations- CICT and LCICT.

Algorithms for implementing CICT and LCICT operations based on calculating the center of the object, selecting its external binary contour, and constructing chords through the center of this object are presented.

The possibilities of CICT and LCICT operations implementation using software and hardware are considered. The structure of a coherent optical processor for image chord transformation is developed. The possibility of realizing integral operations of CICT and LCICT is presented for contoured and complete images based on the calculation of the autocorrelation function of the object image, which is very promising using parallel optical processors.

It is shown that the CICT operation allows achieving invariance to the displacement and rotation of the object, and the LCICT operation to the displacement, rotation, and scale of the object, which will allow applying high-speed algorithms and techniques at the stage of object classification, significantly reducing the volume of computational operations and to implement a real-time mode in image analysis.

In the future, there will be elaborated the multiprocessor systems for invariant object recognition using proposed operations of image chord transformations CICT and LCICT.

\section{References}

1. Jin Lin, Qinzhang Yu, Guibin Chen. Infrared ship target detection based on the combination of Bayesian theory and SVM. Proc. SPIE. 11429, MIPPR 2019: Automatic Target Recognition and Navigation.

2. Yi Wu, Chuangang $\mathrm{Xu}$, Ting Li, Keming Yao, Bing Han. A deep learning-based attribute adaptive infrared maritime ship target detection method. Proc. SPIE 11563, Infrared Device and Infrared Technology. (2020) https://doi.org/ 10.1117/ 12.2579752 .

3. Garcia-Salgado Beatriz, Ponomaryov Volodymyr, REYES-REYES, Rogelio. Parallel artificial neural networks using wavelet-based features for classification of remote-sensing hyperspectral images. Proc. SPIE 11401, Real-Time Image Processing and Deep Learning (2020), doi: 10.1117/12.2556296.

4. Spiliotis Iraklis, Karampasis Nikolaos Boutalis Yiannis. Fast computation of Hahn moments on gray images using block representation. Journal of Electronic Imaging ,Vol. 29, Issue 1 (Feb 2020).

5. Danyu Sun, Jiangming kan, Songnan Chen. Color-based moment invariants under changing illumination. Journal of Electronic Imaging, Vol. 29, Issue 2 (2020).

6. Akbar Naeem, Tehsin Sara, Rehman Haseebur, Rehman Saad, Young Rupert. Hardware design of correlation filters for target detection. Proc. SPIE 10995, Pattern Recognition and Tracking XXX, (2019); doi: 10.1117/12.2519497. 
7. Gardezi A., Malik U., Rehman S., Young R., Birch P., Chatwin C. Enhanced target recognition employing spatial correlation filters and affine scale invariant feature transform. Proc. SPIE 10995, Pattern Recognition and Tracking XXX, (2019); doi: 10.1117/12.2520555.

8. Hoang Thai, Tabbone Salvatore. Invariant pattern recognition using the RFM descriptor. Pattern Recognition, Vol.45, Issue 1, January 2012, Pages 271-284.

9. Guang-Yi Chen, Wen-Fang Xie. Invariant pattern recognition using ring-projection and dual-tree complex wavelets. Proc of ICWAPR International Conference on Wavelet Analysis and Pattern Recognition, 2011, Pages: 182 - 186.

10. Fernández Ariel. Optical architectures for pattern recognition with the generalized Hough transform. Proc. SPIE. 11351, Unconventional Optical Imaging II.

11. Nichol D. Hybrid optical digital computation of global chord functions. Optics Communications, Volume 43, Issue 3, 1 October 1982, Pages 168-170.

12. Casasent D., Chang W. Generalized chord transformation for distortion - invariant optical pattern recognition. Applied Optics, 1983, Vol.21, nr., P.2087-2094.

13. Perju V., Gurau A., Saranciuc D. The optical-electronic pattern recognition system based on the image's logarithmic chord transformation method. In: Optical Pattern Recognition VIII - David P. Casasent, TienHsin Chao, Eds. / Proc SPIE Vol.3073, pp.332-342 (1997). ISBN 9780819424884. 